\title{
A energia eólica como alternativa energética para agricultura familiar em regiões serranas
}

\author{
The wind energy as energetic alternative for family agriculture in mountainous \\ regions
}

\author{
Beatriz Rogers Paranhos, Flávio Castro da Silva e Marcio Cataldi \\ Universidade Federal Fluminense, RJ, Brasil \\ brparanhos@gmail.com; flavioengufla@gmail.com; marcio.cataldi@gmail.com
}

\section{Resumo}

Diante do apelo por medidas de crescimento da participação de energias renováveis no cenário mundial e também da grande dependência brasileira sobre a energia hidráulica, o presente trabalho tem por finalidade provocar o debate sobre a possibilidade de aproveitamento eólico em topos de morros e montanhas, dado o potencial promissor dos ventos nestas regiões. Além disso, existem no Brasil, muitas cooperativas em que o emprego de tecnologias sustentáveis poderia possibilitar a redução dos custos de produção. Desta forma, a Cooperativa Agrícola de Vieira, situada no município de Teresópolis, RJ, foi escolhida para investigação da viabilidade técnica de implantação de aerogeradores em sistema de geração distribuída. Para tal, foram coletados e manipulados os dados de ventos fornecidos pelo Instituto Nacional de Meteorologia (INMET), aplicadas as Leis de Parede para estabelecimento do perfil vertical logarítmico do vento, bem como estimada a produção elétrica do aerogerador nacional selecionado para pesquisa e comparada ao real consumo de uma plantação de referência. Assim, é constatada a capacidade do recurso eólico local em gerar energia elétrica durante todo o ano, contribuindo para o crescimento da utilização da energia eólica em regiões serranas e, consequentemente, para a ampliação da diversificação da matriz elétrica brasileira.

Palavras-chave: Aerogerador; Energia eólica; Vento

\section{Abstract}

Before the appeal for growth measures of renewable energy use in the global scenario and the great Brazilian dependence on hydroelectric energy, this paper intends to open a debate on the capacity of wind energy use in in the top of hills and mountains, considering the promising wind potential in these regions. In addition, there are many cooperatives in Brazil where the use of sustainable technologies could make possible reduce production costs. Therefore, the Agricultural Cooperative of Vieira, located in Teresópolis, a city in the state of Rio de Janeiro, was chosen for a technical feasibility study for the implementation of wind turbines in a distributed generation system. The wind data provided by the National Institute of Meteorology (INMET) was manipulated and the Law of the Wall was applied to establish the logarithmic wind profile, to estimate the electricity production of the national aerogenerator selected and compare the results with the real consumption of a reference field. Thus, it is verified the ability of the local wind resource to generate electricity throughout the year, contributing to increase the use of wind power in mountainous regions and consequently to expand the diversification of the Brazilian energy matrix.

Keywords: Aerogenerator; Wind energy; Wind 


\section{Introdução}

Muitos países vêm estipulando metas e elaborando programas para a criação de um ambiente mais favorável à ampliação do uso de energias renováveis em suas matrizes energéticas. Diante desta conjuntura, destaca-se, dentre as fontes consideradas limpas e renováveis, a energia eólica, denominação dada à energia cinética resultante do deslocamento das correntes de ar na atmosfera (PREMALATHA et al., 2014). A energia eólica é consagrada, sobretudo, pelas baixas emissões, e apenas indiretas, de carbono (ASO et al., 2015).

No Brasil, apesar do representativo avanço na utilização de turbinas eólicas, a energia dos ventos ainda representa uma pequena parcela da produção elétrica total. Segundo a Empresa de Pesquisa Energética (2016), em 2015 a energia hidráulica contribuiu com $64 \%$ de toda a geração elétrica do país, demonstrando uma forte dependência do setor nesta fonte. Enquanto isso, a eólica representou 3,5\% da matriz. Assim, evidencia-se a necessidade de avanços no ramo eólico no que concerne o maior e melhor aproveitamento dos ventos em território brasileiro.

Conforme já descrito por diferentes autores, como, por exemplo, STANGROOM (2004), o escoamento do ar ganha velocidade ao acompanhar a inclinação a montante de morros e montanhas, atingindo seu máximo de intensidade ao chegar ao topo. Desta maneira, os ventos presentes em locais montanhosos apresentam grande potencial de aproveitamento de sua energia.

No caso da Região Serrana do Estado do Rio de Janeiro, Brasil, a agropecuária tem participação significativa no Produto Interno Bruto (PIB), dado o seu clima favorável (IBGE, 2013). Portanto, a utilização de tecnologias sustentáveis, como a aplicação da energia eólica, por comunidades rurais, reflete na possibilidade de redução dos custos de produção relativos ao consumo energético.

Neste contexto, de modo a instigar o maior uso da energia eólica no país em regiões serranas, o presente trabalho tem por finalidade caracterizar e avaliar a capacidade do recurso eólico sobre a área da Cooperativa Agrícola de Vieira (CoopVieira), localizada em Teresópolis - RJ. Além disso, tem como objetivo verificar a eficiência de geradores eólicos de produção nacional, tendo, para isto, sido selecionado o modelo Verne 555 da empresa Enersud.

\section{Materiais e método}

\section{Apresentação da área de estudo}

A Cooperativa Agrícola de Vieira está situada na extensão do km 33 ao km 45 da estrada que liga Teresópolis a Nova Friburgo, a RJ-130, com perímetro de atuação aproximado delimitado na figura 1. Conforme evidenciado pelo Atlas Eólico do Estado do Rio de Janeiro (AMARANTE et al., 2002), a região apresenta ventos predominantemente de quadrante noroeste, norte e nordeste.

O grupo agrícola CoopVieira conta com 53 sócios, sendo 41 agricultores familiares e 12 não agricultores. A produção é voltada para olericulturas em geral, com ênfase nos cultivos de agrião, cebolinha, alface e couve. Apesar de contar com agricultores familiares, o foco da produção agrícola da Cooperativa é industrial, sendo esta intensa durante todo o ano e com elevado gasto energético.

A tabela 1 exemplifica o consumo de uma cultura de 1 ha de agrião da CoopVieira, onde a irrigação representa a única demanda energética. Neste local, um conjunto motobomba trifásica $220 \mathrm{~V}$ de $12,5 \mathrm{HP}$ recalca água para a plantação e, são gastos em média, 21,75 kWh/dia.

Tabela 1 - Acompanhamento do consumo elétrico da cultura de agrião visitada (kWh)

\begin{tabular}{c|c|c|c|c|c|c|c|c|c|c|c|c}
\hline \multicolumn{10}{c}{ Acompanhamento do consumo elétrico da monocultura de agrião $(\mathrm{kWh})$} \\
\hline Média & Jan & Fev & Mar & Abr & Mai & Jun & Jul & Ago & Set & Out & Nov & Dez \\
\hline 505 & 648 & 519 & 456 & 425 & 379 & 375 & 493 & 571 & 553 & 666 & 453 & 530 \\
\hline
\end{tabular}




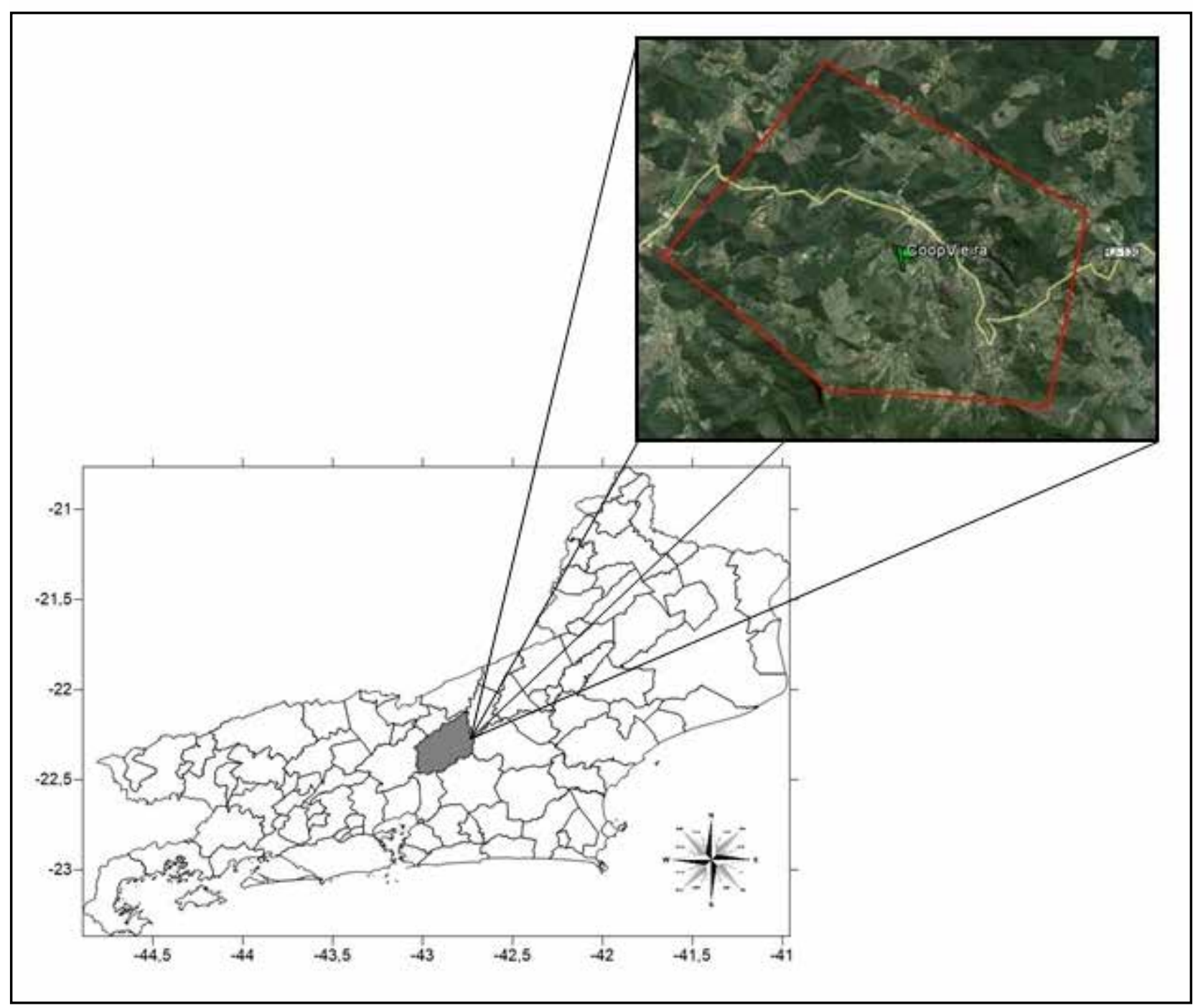

Figura 1 - Perímetro de atuação da CoopVieira Fonte: Adaptado de Google Earth, 2016

Um local favorável, dentro da Cooperativa, para a instalação de aerogeradores se situa nas imediações do Mirante de Vieira, a, aproximadamente, $1149 \mathrm{~m}$ de atitude, com latitude de $-22.277141^{\circ}$ e com longitude de $-42.722884^{\circ}$. Este ponto localiza-se em uma condição de vale, entre duas cadeias montanhosas que formam um corredor de ventos constantes. Além disso, encontra-se em uma área de fácil acesso, facilitando a implantação e manutenção dos equipamentos.

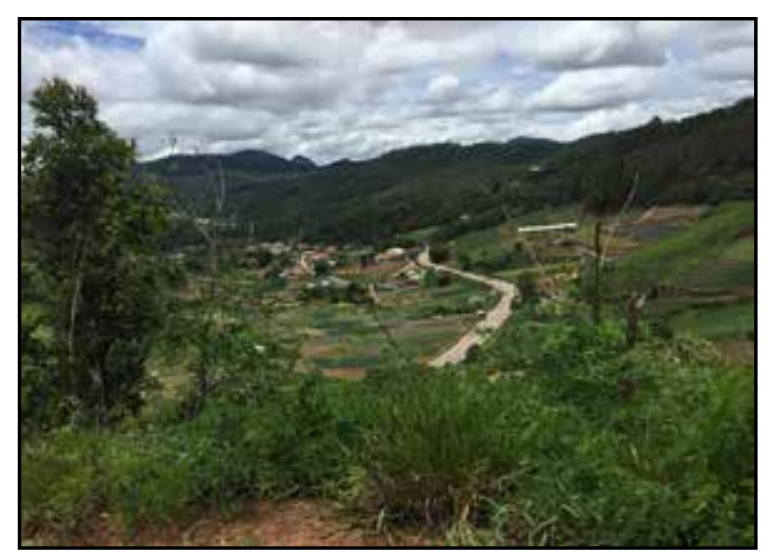

Figura 2 - Vista de Vieira a partir do ponto de interesse para aproveitamento eólico 


\section{Seleção do aerogerador de fabricação nacional}

De modo a valorizar o trabalho do ramo eólico no Brasil, diversas companhias brasileiras foram analisadas e contatadas para conhecimento de seus produtos. Dentre elas, destacou-se pela atenção dada ao projeto, a Enersud ®, empresa com mais de 10 anos de atuação no mercado nacional, situada na cidade de Maricá, Rio de Janeiro. Sua relativa proximidade da zona de estudo também foi considerada.

A Enersud apresenta quatro diferentes modelos de turbinas eólicas. Por meio de debates com a empresa sobre as características do local de interesse de implantação e pela análise das curvas de potência de cada exemplar, o aerogerador modelo Verne 555 foi escolhido, se mostrando o mais adequado ao estudo em questão.

O Verne 555 é um aerogerador de eixo horizontal, com três pás, em configuração upwind. Sua velocidade de partida é de 2,20 m.s-1 e sua potência nominal equivale a $6 \mathrm{~kW}$, atingida a 12,00 m.s-1. Ademais, de maneira a otimizar o aproveitamento do vento, foi selecionada a maior torre fabricada pela empresa, com $18 \mathrm{~m}$ de altura.

A curva de potência, obtida junto aos fornecedores do aerogerador, considera as condições padrão da atmosfera: nível do mar, temperatura de $15 \mathrm{oC}$ e densidade do ar de $1,225 \mathrm{~kg} \cdot \mathrm{m}^{-3}$. Como Teresópolis não se encontra ao nível do mar e, consequentemente, a densidade do ar difere do padrão, então é possível que se tenha uma imprecisão de mais ou menos $5 \%$ no desempenho do equipamento.

\section{Determinação do recurso eólico da área de estudo}

Para caracterização e avaliação da intensidade e comportamento dos ventos próximo à CoopVieira, foram solicitados ao Instituto Nacional de Meteorologia (INMET) os dados horários de vento da estação meteorológica automática mais próxima, a Nova Friburgo - Salinas - A624 (código OMM no 86889). Com uma série temporal iniciando em 17 de setembro de 2010 e finalizando em 30 de novembro de 2015, foram calculadas as médias mensais da velocidade do vento, em m. $\mathrm{s}^{-1}$, e seus respectivos desvios-padrão, também em $\mathrm{m} \cdot \mathrm{s}^{-1}$.

Assim, foi possível estimar o perfil vertical logarítmico do vento. Para tal, foram consideradas as Leis de Parede para uma camada limite atmosférica neutra (STULL, 1988), devido à falta de dados sobre o fluxo de calor na superfície, e também de relações de Businger-Dyer (BUSINGER, 1971) específicas para esta configuração de terreno/atmosfera.

Deste modo, foi estipulado o valor mensal e o valor médio da velocidade de atrito na superfície $u_{\tau} u_{\tau}$ através da equação 1. Por meio da análise do Atlas Eólico do Estado do Rio de Janeiro (AMARANTE et al., 2002), o deslocamento da origem, em que o vento assume velocidade zero, ou seja, o valor do comprimento de rugosidade $z_{0} z_{0}$, foi estimado como sendo $1 \mathrm{~m}$, por se tratar de uma zona pouco urbanizada, porém de relevo montanhoso. A altura de referência $h_{r} h_{r}$ utilizada foi de $10 \mathrm{~m}$, ponto onde foram medidas as velocidades do vento $\overline{u_{x} u_{x}}$. A constante de Von Kármán $k k$ equivale a aproximadamente 0,4 .

$$
u_{\tau}=\frac{k \overline{u_{x}}\left(h_{r}\right)}{\ln \frac{h_{r}}{z_{0}}}
$$

A partir do cálculo de $u_{\tau} u_{\tau}$ foi utilizada a equação 2, que fornece a velocidade do vento em função da altura $(\bar{u}(z) \bar{u}(z))$. Foi possível calcular a velocidade média do vento em diferentes alturas de atuação para cada mês do ano, bem como a sua média a partir dos valores mensais.

$$
\bar{u}(z)=\frac{u_{\tau}}{k} \ln \left(\frac{z}{z_{0}}\right)
$$

As mesmas operações matemáticas (equações 1 e 2) foram feitas a partir dos valores mensais encontrados de desvio padrão. Este procedimento foi realizado para possibilitar a comparação dos valores médios da velocidade do vento somados e subtraídos de 1 desvio padrão (aproximadamente em $68 \%$ de uma distribuição normal), a fim de se definir uma faixa normal de operação para um determinado período e uma determinada altura de interesse.

\section{Produção energética estimada e fator de capacidade do aerogerador escolhido}

Para estimativa da probabilidade de ocorrência de uma dada velocidade do vento no local de interesse e o consequente cálculo da produção potencial de energia para cada mês do ano, a Distribuição de Weibull foi adotada (MATHEW, 2006). Nesta distribuição, as variações na velocidade do vento são caracterizadas por duas funções, 
sendo estas a função de densidade de probabilidade e a função de distribuição cumulativa (MATHEW, 2006). O estudo em questão empregou apenas a primeira delas. A função de densidade de probabilidade $\left(f(u) f_{f(u)}\right)$ indica a fração do tempo para a qual o vento se encontra a uma dada velocidade uu e é representada pela equação 3 .

$$
f(u)=\frac{k}{c}\left(\frac{u}{c}\right)^{k-1} e^{-\left(\frac{u}{c}\right)^{k}}
$$

O fator de forma $k_{k}$ foi obtido através da análise do Atlas Eólico do Estado do Rio de Janeiro (AMARANTE et al., 2002), com valor igual a 2. Neste caso, deriva-se a Distribuição de Rayleigh, caracterizada apenas pelo fator de escala $c c\left(\mathrm{em} \mathrm{m} . \mathrm{s}^{-1}\right)$. O fator de escala foi encontrado por meio da equação 4 , em que $\Gamma$ representa a função gama.

$$
c=\frac{\bar{u}}{\Gamma\left(1+\frac{1}{k}\right)}
$$

Desta maneira, foram calculados os fatores de escala mensais a $18 \mathrm{~m}$ de altura e em três configurações distintas: utilizando, nestas elevações, a velocidade média do vento, sua velocidade média somada a 1 desvio padrão e sua velocidade média subtraída a 1 desvio padrão. Ou seja, a intenção era criar funções de densidade de probabilidade para cada um destes possíveis cenários.

Associando a curva de potência do aerogerador $P(u) P(u)$, em kW, elemento caracterizador da eficiência energética do equipamento, com a função de densidade de probabilidade $f(u) f(u)$, em \%, responsável por evidenciar o comportamento do vento em um local especificado, é possível determinar a produção energética $P E P E$, em kWh, em um certo período de tempo, como indicado pela equação 5.

$$
P E=H \int_{u_{i}}^{u_{f}} P(u) f(u) d u \cong H \sum_{i=1}^{N} P\left(u_{i}\right) f\left(u_{i}\right) \Delta u_{i}
$$

No presente trabalho, o objetivo foi encontrar a produção energética mensal, sendo portanto, $H H$ igual ao número de horas contidas em um mês. A determinação da $P E$ foi feita para a altura de $18 \mathrm{~m}$, considerando a velocidade média do vento, a velocidade média somada a 1 desvio padrão e a velocidade média subtraída a 1 desvio padrão.

Por fim, foi definido o fator de capacidade $F C_{F C}$ da turbina eólica Verne 555, representando a razão entre a energia efetivamente gerada para as circunstâncias locais e a energia teórica que seria produzida considerando-se a potência nominal $P_{n} P_{n}$, em $\mathrm{kW}$, da máquina, conforme a equação 6 . Nesta condição foi empregada a produção anual energética $P A E_{P A E}$, sendo obtida por meio da equação 5, quando $H H$ equivale ao número de horas contidas em um ano. Novamente, o cálculo foi realizado para as três configurações de vento anteriormente mencionadas, para uma torre de sustentação de $18 \mathrm{~m}$ de altura.

$$
F C=\frac{P A E}{8760 P_{n}}
$$

\section{Resultados e discussão}

A determinação da velocidade do vento em função da altura é um fator-chave para a verificação do potencial aproveitamento da energia eólica em um certo local. O gráfico da figura 3 representa o perfil vertical dos campos de velocidade de vento média calculados a partir dos dados da estação meteorológica de Nova Friburgo - Salinas - A624. O eixo das abcissas reproduz as diferentes velocidades médias que o vento é capaz de possuir em função de sua altura, simbolizada pelo eixo das ordenadas. Observa-se que existe grande variação da velocidade em função da altura na faixa entre 0 e 40 m, quando, então, o incremento na velocidade começa a variar de forma menos significativa e mais regular.

No caso do aerogerador Verne 555, a altura de captação dos ventos é feita a 18 m da superfície. Pode-se analisar, através da figura 4, a variabilidade anual da velocidade do vento a esta altura, sendo a linha em laranja o comportamento médio do vento e as barras de erro correspondentes aos respectivos desvios-padrão. Em termos médios, é verificado baixa disparidade para os valores de velocidade nesta região ao longo do ano. O mês de pico é repre- 


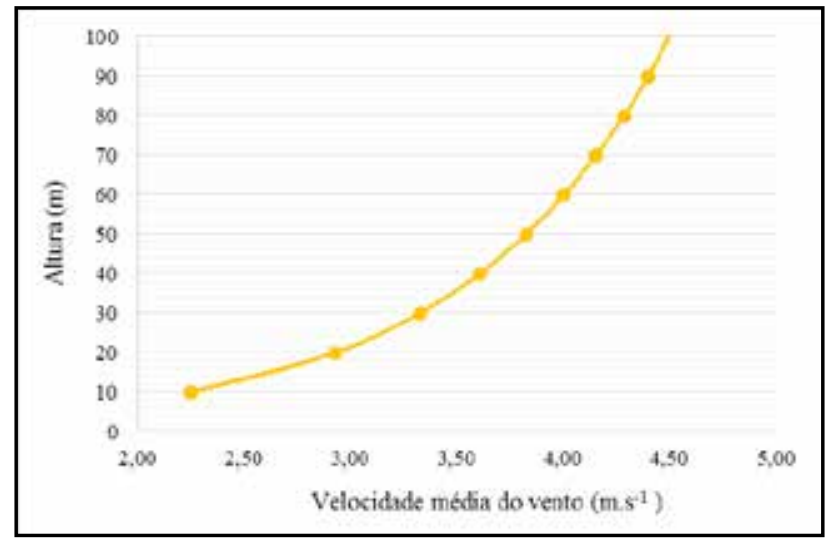

Figura 3 - Perfil vertical do vento da área de estudo

sentado por outubro, com 3,32 m.s-1 a $18 \mathrm{~m}$. Já fevereiro se classifica como o período de ventos mais fracos, com 2,46 m.s-1 a 18 m. Dado que a velocidade de arranque do Verne 555 é de 2,20 m.s-1, sendo um valor característico para diversos modelos, esta turbina eólica seria capaz de dar início ao seu funcionamento, assim como mantê-lo durante o ano, na área de estudo.

Para que seja possível atingir a potência nominal do aerogerador em questão, na faixa de $6 \mathrm{~kW}$ ao nível do mar, o vento deve estar a uma velocidade de aproximadamente $12 \mathrm{~m} . \mathrm{s}-1$, o que corresponde a 43,2 km.h-1. Na área de estudo, este valor seria atingido, em condições médias, acima da altura da camada limite atmosférica, que pode medir até cerca de $3000 \mathrm{~m}$ (STULL, 1988). Vale ressaltar que o modelo se enquadra no grupo de aeroturbinas de pequeno porte, o que enfatiza a impraticabilidade de se chegar ao seu máximo valor de potência.

Além disso, é de suma importância a avaliação da produção elétrica do aerogerador e sua comparação à demanda local, para, de fato, averiguar a eficiência do equipamento escolhido para a realidade da região. Desta forma, a figura 5 equipara a geração de energia elétrica mensal estimada do Verne 555, a $18 \mathrm{~m}$ de altura, para os ventos medidos pela estação meteorológica mais próxima, com o consumo energético da tabela 1 , tendo sido esta lavoura considerada como referência para o estudo. Dentro da faixa normal de operação do modelo de turbina eólica escolhido, a linha em azul simboliza a energia produzida considerando as velocidades médias do vento somadas aos desvios-padrão associados, a linha em verde considera velocidades médias e a linha em magenta refere-se às velocidades médias subtraídas aos desvios-padrão. Já o traço em preto representa o consumo da plantação.

Nota-se, pela figura 5, que o único cenário capaz de suprir as necessidades energéticas da cultura é aquele referente à eletricidade fornecida em casos de velocidades médias somadas ao desvio padrão, a partir de meados de março até dezembro. Desta forma, um aerogerador de $6 \mathrm{~kW}$ de potência nominal a $18 \mathrm{~m}$ de altura não conseguiria suprir totalmente a produção de uma monocultura da Cooperativa Agrícola de Vieira como a analisada. Examinando as velocidades médias a $18 \mathrm{~m}$ da superfície, chega-se a uma média de produção de $124,05 \mathrm{kWh}$ por mês, o que colabora com cerca de $25 \%$ do gasto médio mensal de $505 \mathrm{kWh}$ da lavoura. Para atender com segurança a necessidade energética atual durante o ano todo apenas desta plantação, seriam necessários cerca de 7 aerogeradores deste modelo com torres de sustentação de $18 \mathrm{~m}$.

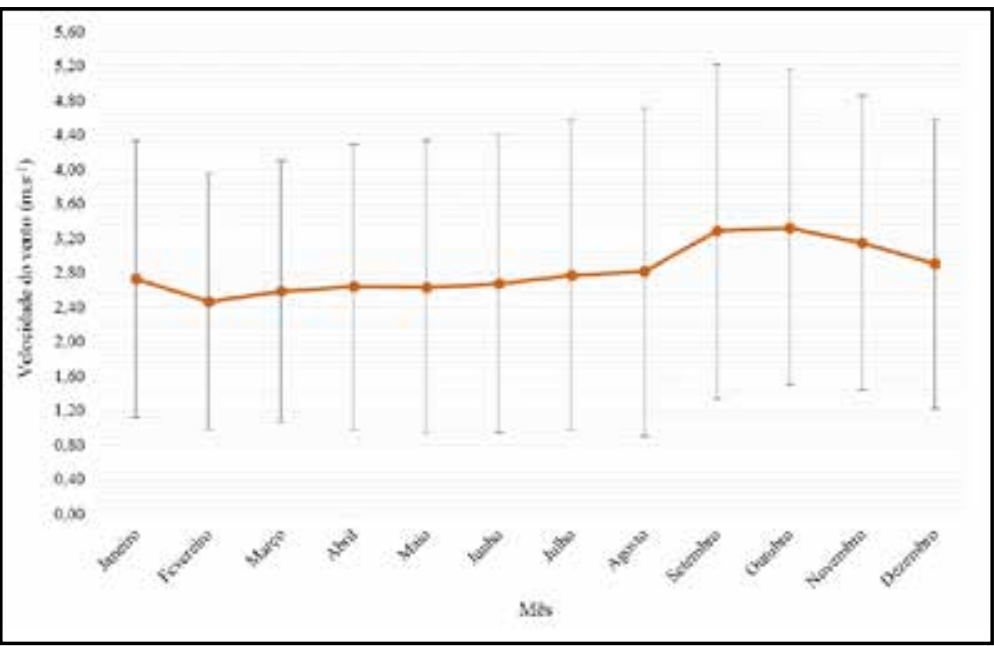

Figura 4 - Comportamento mensal da velocidade do vento a $18 \mathrm{~m}$ de altura 


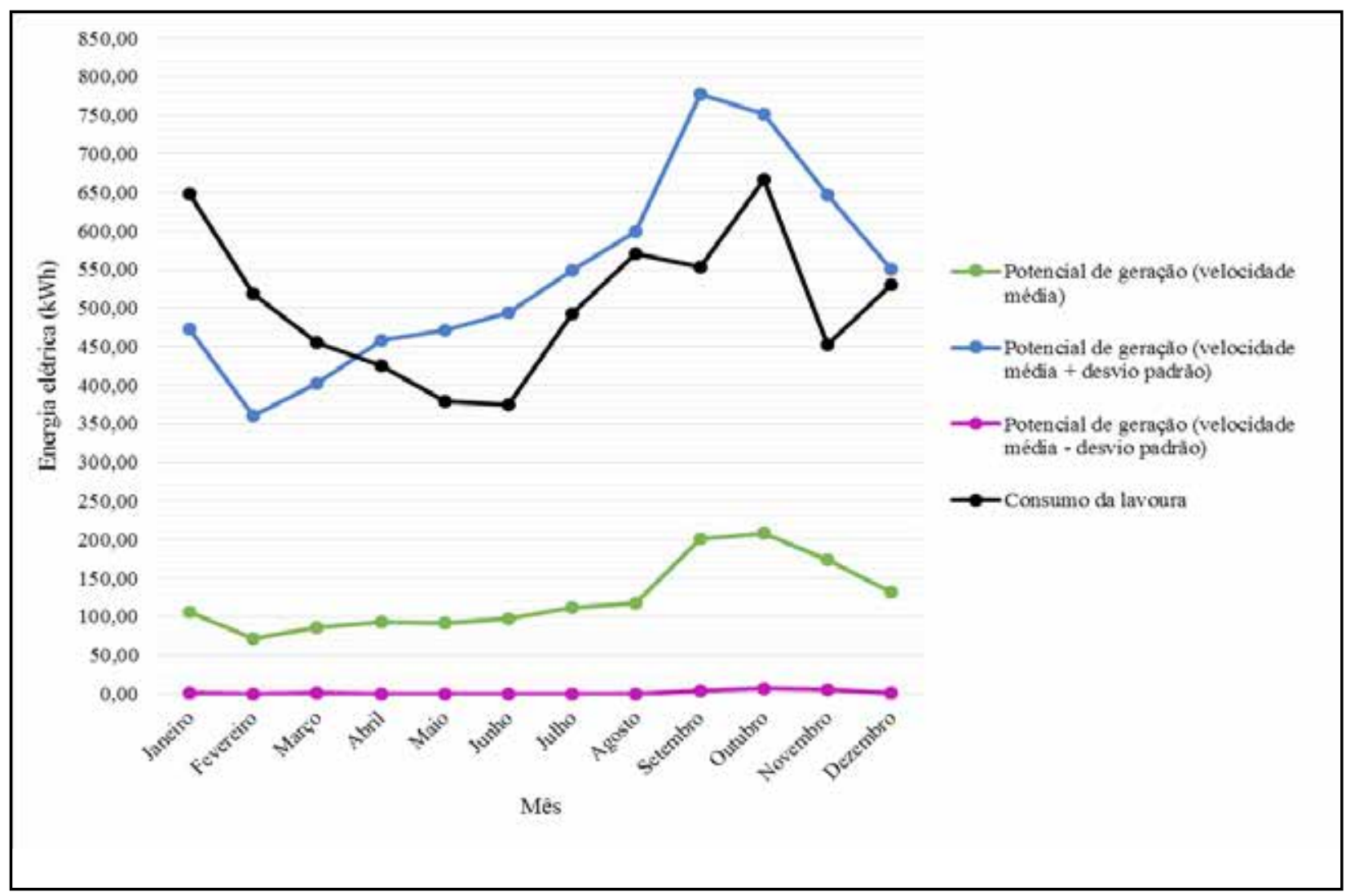

Figura 5 - Produção potencial de energia elétrica do Verne 555 a 18 m de altura

Observando os fatores de capacidade do Verne 555 dentro destas três configurações, conforme a tabela 2, percebese que, no melhor dos casos, ou seja, considerando o cenário de vento na região como sendo o seu valor médio mais um desvio padrão, o equipamento aproveita 12 \% de sua potência nominal. Este valor, segundo Wiser et al. (2012), é considerado expressivo para um aerogerador de pequeno porte em condições de vento de pouca intensidade. Desta forma, é importante ressaltar que este índice é função da velocidade do vento no local, possuindo valores mais elevados em áreas com velocidades médias de vento superiores às encontradas nesta região. É esperado que, em localidades de maior altitude, assim como em maiores alturas, esta velocidade média do vento também seja mais elevada (MATHEW, 2006). No entanto, como a região não possui registros de vento, por exemplo, nos picos dos morros, somente a partir da utilização da modelagem numérica isso poderia ser avaliado.

Tabela 2 - Fator de capacidade do Verne 555 em diferentes configurações de vento a 18 m de altura

\begin{tabular}{c|c|c}
\hline \multicolumn{3}{c}{ Fator de capacidade } \\
\hline $\begin{array}{c}\text { Velocidade } \\
\text { média }\end{array}$ & $\begin{array}{c}\text { Velocidade } \\
\text { média + desvio } \\
\text { padrão }\end{array}$ & $\begin{array}{c}\text { Velocidade } \\
\text { média - desvio } \\
\text { padrão }\end{array}$ \\
\hline 0,03 & 0,12 & 0,00 \\
\hline
\end{tabular}

Supondo o emprego de um aerogerador de padrão internacional, com, por exemplo, $120 \mathrm{~m}$ de torre e potência nominal de 4,5 MW, as velocidades médias mensais do vento nesta altura seriam da ordem de 4 a 5 m.s- 1 na região e produziriam na faixa de $500 \mathrm{~kW}$. Este valor, considerando um mês de consumo e uma hora de funcionamento ao dia, corresponderia a, aproximadamente, $15000 \mathrm{kWh}$ e seria capaz de suprir a demanda média de cerca de 29 plantações semelhantes à avaliada neste estudo. Assim, uma turbina eólica deste porte seria capaz de abastecer boa parte dos 41 agricultores da CoopVieira e seu atual modelo de produção. Todavia, cabe destacar que os custos de aquisição, implementação, operação e manutenção de um aerogerador de padrão internacional superam em cerca 
de quatro ordens de grandeza, ou mais, os mesmos custos do Verne 555, de produção nacional, o que inviabilizaria a sua implantação.

De qualquer forma, independente da eficiência da turbina, a disposição do sistema eólico conectado à rede elétrica em modo de geração distribuída, conforme as Resoluções Normativas no 482 (2012) e no 687 (2015) da Agência Nacional de Energia Elétrica (ANEEL), demonstra uma alternativa interessante aos agricultores. Nesta situação, toda a energia ativa gerada pelos aerogeradores que não seja consumida é disponibilizada à rede e pode ser convertida em créditos de energia $(\mathrm{kWh})$, representando uma redução nos custos de produção dos mesmos.

\section{Conclusões}

Os dados meteorológicos medidos pela estação de Nova Friburgo - Salinas indicam que, a 18 m da superfície, o vento na região assume velocidade suficiente ao longo do ano para operação de aerogeradores, evidenciando a capacidade de aproveitamento da energia eólica em regiões serranas. Ademais, existem pontos dentro da Cooperativa de fácil implantação da tecnologia. Por outro lado, o aerogerador Verne 555, por operar na altura de $18 \mathrm{~m}$, onde o vento na região não possui intensidade muito significativa, não apresentou potência suficiente para suprir o consumo elétrico de uma monocultura de agrião de 1 ha da CoopVieira.

Sendo assim, um aerogerador de $6 \mathrm{~kW}$ de potência nominal com $18 \mathrm{~m}$ de altura permitiria reduzir a dependência da energia disponibilizada pelo distribuidor, em sua maioria hidráulica e termoelétrica, mas não seria capaz de tornar o produtor totalmente independente da rede sistêmica de energia. Esta contribuição poderia expandir-se com a otimização de práticas mais racionais e sustentáveis de consumo. Outra possibilidade para a área de estudo seria a aplicação de um aerogerador que operasse em alturas mais elevadas, onde o vento tende a ser mais intenso, ou mesmo a avaliação de outros pontos próximos da cultura onde o vento possua maior intensidade. Uma última alternativa seria a instalação de aerogeradores de grande porte, porém, isto implicaria em despesas financeiras muito maiores para aquisição, instalação, operação e manutenção do mesmo.

Por fim, este artigo evidencia que com a tecnologia, a altura de operação e a capacidade de geração dos aerogeradores destinados a microgeração que existem no mercado nacional ou internacional, é necessário que sejam feitos estudos detalhados da distribuição espacial dos recursos de vento disponíveis em cada região. Dada a dificuldade em se obter registros de séries históricas destes dados, evidencia-se a importância da modelagem numérica para uma melhor reprodução do comportamento do vento sobre toda a área de interesse, proporcionando resultados mais representativos para este tipo de análise e auxiliando na escolha espacial de locais de instalação de aerogeradores.

Desta forma, acredita-se que seja realmente possível a aplicação da energia eólica em topos de morros e montanhas no Brasil. Além de ser uma fonte de energia inesgotável e contribuir para a diversificação da matriz elétrica, a energia eólica, quando planejada adequadamente, é bem menos nociva ao meio ambiente e à sociedade. Portanto, é de total relevância o investimento nesta forma promissora de energia para além do litoral brasileiro, contribuindo para sua evolução. O Brasil é um país de grande território com capacidade de exploração de seus recursos em toda sua extensão.

\section{Agradecimentos}

Agradecimentos à Cooperativa Agrícola de Vieira, CoopVieira, e à empresa Enersud, pela atenção e informações fornecidas, tão essenciais para a realização do projeto em estudo.

\section{Referências}

AMARANTE, O. A. C.; SILVA, F. J. L. da; RIOS FILHO, L. G. Atlas eólico do Estado do Rio de Janeiro. Secretaria de Estado de Energia, da Indústria Naval e do Petróleo. Governo do Estado do Rio de Janeiro. Rio de Janeiro. 2002.

ASO, R.; CHEUNG, W. M. Towards greener horizontal-axis wind turbines: analysis of carbon emissions, energy and costs at the early design stage. Journal of Cleaner Production, v. 87, p. 263-274, 2015.

BRASIL. Resolução Normativa ANEEL no 482, de 17 de abril de 2012. Estabelece as condições gerais para o acesso de microgeração e minigeração distribuída aos sistemas de distribuição de energia elétrica, o sistema de compensação de energia elétrica, e dá outras providências. Diário Oficial [da] República Federativa do Brasil, Brasília, DF, p. 53, 19 abr. 2012. Seção 1. 
BRASIL. Resolução Normativa ANEEL no 687, de 24 de novembro de 2015. Altera a Resolução Normativa no 482, de 17 de abril de 2012, e os Módulos 1 e 3 dos Procedimentos de Distribuição - PRODIST. Diário Oficial [da] República Federativa do Brasil, Brasília, DF, no 230, p. 45, 2 dez. 2015. Seção 1.

BUSINGER, J. A., WYNGAARD, J. C., JZUMI, Y., BRADLEY, E. F., FLUX. Profile relationships in the Atmospheric Surface Layer. Journal of the Atmospheric Sciences, vol. 28, pp. 181-189, 1971.

EMPRESA DE PESQUISA ENERGÉTICA. Balanço Energético Nacional 2016 - Ano base 2015. Rio de Janeiro, RJ, 2016. 292 p.

INSTITUTO BRASILEIRO DE GEOGRAFIA E ESTATÍSTICA. Cidades - Teresópolis. Disponível em: <http://cod. ibge.gov.br/B6B>. Acesso em: 11 fevereiro 2016.

MATHEW, Sathyajith. Wind energy: fundamentals, resource analysis and economics. Berlin: Springer, 2006.

PREMALATHA, M.; ABASSI, T.; ABASSI, T.; ABASSI, S.A. Wind energy: Increasing deployment, rising environmental concerns. Renewable and Sustainable Energy Reviews, v. 31, p. 270-288, 2014.

STANGROOM, P. CFD modelling of wind flow over terrain. Tese de Doutorado. University of Nottingham, 2004. STULL, Roland B. An introduction to boundary layer meteorology. Atmospheric Sciences Library, Dordrecht: Kluwer, 1988, v. 1, 1988.

WISER, R., LANTZ, E., Bolinger, M., BOLINGER, M., HAND M. (2012). Recent developments in the levelized cost of energy from U.S. wind power projects. International Energy Agency. 2012. 